\title{
Usabilidade e Adaptabilidade Web: Uma proposta de Inovação de um Sistema de Apoio à Gestão Escolar
}

\author{
Sandro Marques de Carvalho ${ }^{1}$, Eudis Oliveira Teixeira ${ }^{2}$ \\ ${ }^{1}$ Faculdade de Ciências Aplicadas e Sociais de Petrolina (FACAPE) \\ 56328-903 - Petrolina - PE - Brazil \\ ${ }^{2}$ Instituto Federal do Sertão Pernambucano (IF SERTÃO-PE) \\ 56314-520 - Petrolina - PE - Brazil \\ sandro.marques.comp@gmail.com, eudis.oliveira@ifsertao-pe.edu.br
}

\begin{abstract}
This paper describes the analysis of usability the adaptability of the Support System for School Management - SAGE, based on principles and usability goals and Responsive Web Design, which allows navigation of websites from a variety of devices. Were used questionnaires to users of the system, analysis of the current interface based on usability principles and goals and those arising from the experience with users, prototypes in the medium and low fidelity, and usability testing with users. From the results of the analysis found that the degree of difficulty and satisfaction of users in the current system and presented a new proposal to interface usable and adaptable to the System.
\end{abstract}

Keywords: Interaction Design, Web Usability, Adaptability Web, Prototyping, Responsive Web Design.

Resumo. Este artigo descreve a análise de usabilidade e adaptabilidade do Sistema de Apoio à Gestão Escolar - SAGE, com base em princípios e metas de usabilidade e na técnica Responsive Web Design, que permite a navegação de websites a partir de uma variedade de dispositivos. Foram utilizados questionários aplicados a usuários do sistema, análise da interface atual baseada em princípios e metas de usabilidade e as decorrentes da experiência com os usuários, protótipos de baixa, média e alta fidelidade, além de testes de usabilidade com os usuários a partir dos protótipos criados. Através dos resultados da análise encontrou-se o grau de dificuldade e satisfação dos usuários no atual sistema e apresentou-se uma nova proposta usável e adaptável de interface para o Sistema.

Palavras-chave: Design de Interação, Usabilidade web, Adaptabilidade web, Prototipação, Web Design Responsivo.

\section{Introdução}

A utilização de dispositivos móveis para acesso a internet vem crescendo consideravelmente. Com o crescimento do número de dispositivos no mercado, nota-se a dificuldade em se adequar a visualização do conteúdo nos diversos tamanhos de tela e resoluções dos dispositivos, o que torna a navegação desgastante e limitada. 
Em resposta a essa necessidade, versões móveis dos sites foram adaptadas. Os desenvolvedores procuravam detectar o meio pelo qual o usuário estava acessando e redirecionavam o acesso para um código cuja versão seria mais adequado. Apesar dos sistemas adaptativos e personalizados adaptarem informações com base nas características do perfil do usuário, muitos apresentam a interface e a interação com o usuário sem considerar o dispositivo, a plataforma e a resolução de tela do usuário. Este é um problema atual, visto os inúmeros dispositivos e resoluções não padronizados [6].

A adaptabilidade é uma qualidade particularmente esperada em sistemas em que o público-alvo é vasto e variado. Nestes casos, fica evidente que uma única interface não pode atender plenamente a todos os diferentes tipos de usuários. Dois subcritérios participam da adaptabilidade: a flexibilidade e a consideração da experiência do usuário [1]. Chamadas dentro do design de interação de metas decorrentes da experiência do usuário, [4] ressaltam que o objetivo de desenvolver produtos interativos, agradáveis, divertidos e esteticamente apreciáveis está principalmente na experiência que estes proporcionarão ao usuário, isto é, como o usuário se sentirá na interação com o sistema.

Segundo [4], a usabilidade é frequentemente considerada como um elemento que proporciona que uma aplicação pode possuir facilidades, eficiência e agilidade para o usuário que está utilizando a aplicação. Tem sido objeto de estudo de uma área multidisciplinar que atualmente está em foco: a Interação Humano-Computador (IHC).

Tendo em vista as soluções tradicionalmente utilizadas, este trabalho propõe realizar uma análise de usabilidade através de metas decorrentes da experiência dos usuários de um sistema de apoio à gestão educacional, assim como abordar a adaptabilidade deste sistema a partir da utilização do Responsive Web Design (RWD), uma técnica que permite a navegação de websites a partir de uma variedade de dispositivos e resoluções [2].

\section{Responsive Web Design}

O termo "Responsive Web Design" foi utilizado e explicado pela primeira vez em 2010 por Ethan Marcotte [2]. Para o autor, responsive é uma técnica que permite a navegação de websites a partir de uma variedade de dispositivos, desde smartphones e tablets a computadores desktop, ajustando a sua estrutura ao tamanho da janela de visualização, de forma a proporcionar uma boa experiência de utilização no maior número de dispositivos possível.

Para tornar viável o desenvolvimento em RWD, três tecnologias, conhecidas por "trinca tecnológica do web design responsivo" [10], são essenciais: Layout Fluído, Imagens e mídias flexíveis e Media queries.

No Layout fluído, as medidas CSS (Cascading Style Sheets) são definidas em unidades relativas, tais como porcentagens, e não em medidas absolutas, como pixel e mm (milímetro). Imagens e mídias flexíveis são aquelas capazes de contrair ou expandir suas dimensões, ou ter suas dimensões alteradas por scripts em função do contexto onde são renderizadas. As mídias mais comuns presentes em layouts são: vídeos, áudio e widgets em geral. Media queries é uma tecnologia CSS que permite o uso de folhas de estilos baseadas em determinadas características do dispositivo ao qual o layout será exibido. 
Segundo [9], RWD não diz respeito simplesmente e somente à adaptação do layout ao tamanho da tela, o conceito de design responsivo na sua forma ampla, deve ser entendido como o design capaz de "responder" às características do dispositivo ao qual é servido. Responder, nesse contexto, tem o sentido de movimentar-se expandindo e contraindo. Em resumo, o design responsivo expande e contrai a visualização da interface com a finalidade de fornecer uma experiência agradável ao usuário, do ponto de vista da leitura e navegação independentemente do dispositivo utilizado.

\section{Usabilidade em Sistemas Educacionais}

Para Nielsen [3], o termo usabilidade é utilizado para medir a qualidade das interfaces. Está relacionada com a facilidade de aprendizado, eficiência, memorização, tolerância a erros e a satisfação do usuário. Há diversos problemas relacionados com a usabilidade de produtos ou sistemas, estes estão diretamente ligados ao quanto desses sistemas são realmente fáceis e agradáveis de usar e aprender.

Conforme proposto na introdução, este trabalho objetiva inicialmente realizar uma análise de usabilidade de um sistema de apoio à gestão educacional, através de protótipos de baixa e média fidelidade, tendo como base o referencial teórico/metodológico da área de conhecimento Interação Humano Computador (IHC), apresentando um design adequado a um ambiente educacional web através de uma interface de uso simples, de fácil aprendizado, tolerante a erros, esteticamente agradável e que satisfaça aos usuários (Preece e Rogers, 2005) [4].

\subsection{Sistema de Apoio à Gestão Educacional - SAGE}

O SAGE é um software livre de gestão escolar, desenvolvido por pesquisadores de um Instituto Federal de Educação, Ciência e Tecnologia. Teve o início do ciclo de vida em 2006 a partir da necessidade de desenvolver e implantar uma aplicação web que desse suporte aos diversos setores educacionais da instituição, no entanto, durante o processo de desenvolvimento não foram observados e nem levados em consideração os usuários que teriam dificuldade de acesso às informações, com isso, não foram seguidas orientações e recomendações para a garantia da usabilidade de seus usuários. Interface do sistema atual é apresentada através da Figura 1.

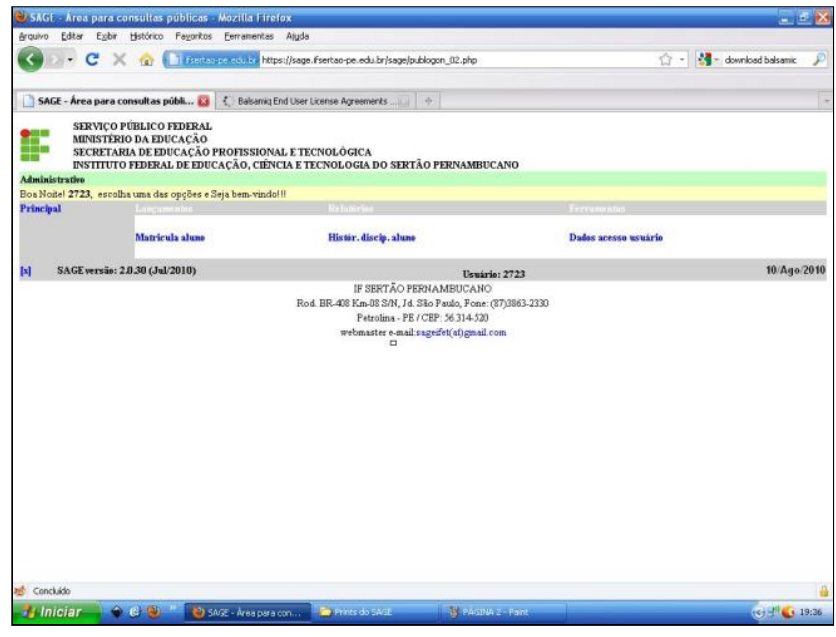

Figura 1: Página de apresentação das opções para o Aluno. 
Com base na realidade apresentada acima, é importante contribuir significantemente para a melhoria da interface atual do sistema permitindo que alunos e servidores possam interagir de forma eficiente em um ambiente simples e fácil de aprender e utilizar, além de permitir uma boa experiência de visualização, fácil leitura e navegação para uma ampla gama de dispositivos.

\section{Metodologia}

Para atender aos objetivos da pesquisa, utilizou-se neste trabalho um processo de obtenção de princípios e requisitos de usabilidade, que tem sido confirmado como uma fase relevante para identificar, refinar e especificar as necessidades em um processo de Software [5]. Para realização deste procedimento foram utilizadas as seguintes etapas:

- Aplicação de questionários;

- Analise do sistema atual com base em princípios e metas de usabilidade e as decorrentes da experiência com os usuários;

- Prototipação e;

- Testes de usabilidade.

Com a aplicação dos questionários buscaram-se subsídios para definição de requisitos e atributos de usabilidade, através das informações acerca das facilidades, dificuldades e necessidades dos usuários do SAGE.

Em seguida foi realizada a análise da interface atual do sistema SAGE no intuito de propor mudanças. A referência teórica utilizada para análise foram os princípios e as Metas de Usabilidade e as Decorrentes da experiência com os usuários definidos em literatura de Design de Interação como eficientes formas de avaliação e análise de interfaces de produtos ou sistemas [4], preocupando-se em preencher critérios específicos de usabilidade e em explicar a qualidade da experiência.

Com base nos requisitos e princípios de usabilidade identificados, foram gerados os protótipos de baixa fidelidade utilizando paper prototype uma técnica formal e informal para o desenvolvimento, demonstração e avaliação do design de interfaces de usuários, que dá suporte a iterações rápidas, gerando modelos preliminares do ambiente proposto para testes e análise de resultados [7]. Após a prototipação das telas em baixa fidelidade, estas foram aperfeiçoadas e refinadas utilizando a prototipação em média fidelidade, utilizando para isso, os softwares balsamiq ${ }^{1}$ e pencil ${ }^{2}$, específicos para este tipo de tarefa.

Por fim, empregando os protótipos gerados, foram realizados testes de usabilidade com o objetivo de validar os requisitos identificados na pesquisa. Estes testes envolveram avaliar o desempenho de usuários na realização de tarefas, em condições controladas de laboratório com o objetivo de obter dados que mostrassem a facilidade de uso e capacidade de aprendizado de um produto ou sistema, combinando técnicas de observação, questionários e testes com usuários [4].

\footnotetext{
${ }^{1}$ Balsamiq - Software para prototipação em média fidelidade. http://www.balsamiq.com

${ }^{2}$ Pencil - Software para prototipação em média fidelidade. http://www.pencil.com
} 
Após os testes de usabilidade foi utilizado o framework Bootstrap ${ }^{3}$ para o desenho das interfaces responsivas, de forma a permitir a navegação do sistema de apoio à gestão educacional a partir de uma variedade de dispositivos, desde smartphones e tablets a computadores desktop, ajustando a sua estrutura ao tamanho da janela de visualização. Segundo [8], o Bootstrap é um conjunto de recursos contendo diversos componentes web para desenvolver aplicações web/mobile de forma mais fácil e objetiva. Segundo o autor, esta aplicação tem como meta tornar o desenvolvimento de interfaces responsivas mais simples.

\section{Resultados}

\subsection{Aplicação dos Questionários}

Foram enviados questionários a 30 (trinta) potenciais usuários (servidores e alunos), com idade entre 15 e 50 anos, nível médio (completo ou não) ou superior (completo ou não), mais de um ano de conhecimentos básicos de informática e de utilização de aplicativos básicos web. A princípio, pelo número de 15 (quinze) dos questionários respondidos, imaginou-se que seria insuficiente para obter resultados para pesquisa, porém, pelo teor e qualidade das respostas e, sobretudo pelo perfil dos que responderam, considerou-se importante para esta fase do projeto.

Um importante aspecto observado nas respostas é a dificuldade de localização dentro do sistema, bem como, o excesso de informações que na visão dos questionados são desnecessárias. Cerca de 50\% dos entrevistados apresentaram esses fatores como principais erros de interface. Outro fator preponderante foi que $70 \%$ dos entrevistados tinham pequenas dificuldades de interação com o sistema em tipos específicos de falta de qualidade nas interfaces como: dificuldades de aprendizado, dificuldades de memorização e sentimento desagradável quanto ao aspecto visual do sistema. Alguns admitem que tais dificuldades de usabilidade estabeleçam sérias restrições ao desenvolvimento das atividades de interação dos usuários com o sistema.

\subsection{Análise do Sistema Atual}

As tabelas a seguir mostram os resultados da análise de usabilidade referentes ao design e princípios de usabilidade (Tabela 1), metas de usabilidade (Tabela 2) e metas decorrentes da experiência com o usuário (Tabela 3).

\begin{tabular}{|l|l|c|l|l|}
\hline \multicolumn{2}{|l|}{ Design e princípios de Usabilidade } \\
\hline ID & Princípios & Atende & Não Atende & Descrição \\
\hline 1 & Visibilidade & & $\mathrm{X}$ & Dificuldades na visualização de links e botões \\
\hline 2 & Feedback & & $\mathrm{X}$ & $\begin{array}{l}\text { O Sistema deveria mostrar ao usuário onde ele está e o } \\
\text { que ele já fez, oferecendo a opção voltar. }\end{array}$ \\
\hline 3 & Mapeamento & & $\mathrm{X}$ & $\begin{array}{l}\text { Existem alguns botões de redefinir sem função alguma } \\
\text { que ficam juntos aos botões avançar ou concluir, isso } \\
\text { pode atrapalhar o usuário. }\end{array}$ \\
\hline 4 & Consistência & $\mathrm{X}$ & & Boa consistência para realizar tarefas, as operaç̃̃es são \\
\hline
\end{tabular}

\footnotetext{
${ }^{3}$ Bootstrap - Framework para desenvolvimento web responsivo. http://getbootstrap.com
} 


\begin{tabular}{|l|l|l|l|}
\hline 5 & Affordance & $\mathrm{X}$ & semelhantes para os diferentes tipos de usuários. \\
\hline & $\begin{array}{l}\text { Botões como avançar e concluir são bem visíveis e } \\
\text { chamativos, porém, existem alguns itens que o usuário } \\
\text { não percebe que deve alterá-los, como por exemplo, } \\
\text { escolher se quer visualizar as notas da unidade, do ano ou } \\
\text { todo o histórico, essa opção de escolha não é percebida } \\
\text { pelo usuário. }\end{array}$ \\
\hline
\end{tabular}

Tabela 1: Análise do design e princípios de Usabilidade.

\begin{tabular}{|l|l|c|c|l|}
\hline \multicolumn{2}{|l|}{ Metas de Usabilidade } & Atende & Não Atende & Descrição \\
\hline ID & Metas & $\mathrm{X}$ & & Cumpre as tarefas esperadas \\
\hline 1 & Eficácia & & $\mathrm{X}$ & $\begin{array}{l}\text { Erros de design, excesso de informações, cores são muito } \\
\text { contrastantes, botões desnecessários e sem funções. }\end{array}$ \\
\hline 2 & Eficiência & $\mathrm{X}$ & $\begin{array}{l}\text { Localização próxima de botões como Redefinir e } \\
\text { Concluir, não utiliza janelas de confirmação. }\end{array}$ \\
\hline 3 & Segurança & $\mathrm{X}$ & $\mathrm{X}$ & $\begin{array}{l}\text { Interação on-line entre servidores e alunos realizadas de } \\
\text { forma eficaz. }\end{array}$ \\
\hline 4 & Utilidade & $\begin{array}{l}\text { Muitas telas para atingir objetivos, muitas informações } \\
\text { agrupadas, códigos não facilmente compreendidos e falta } \\
\text { de informações de ajuda. }\end{array}$ \\
\hline 5 & $\begin{array}{l}\text { Capacidade de } \\
\text { Aprendizado }\end{array}$ & $\begin{array}{l}\text { Muitas telas com muitos estágios para atingir objetivos e } \\
\text { sistema de matrícula considerado complexo por ser } \\
\text { utilizado esporadicamente. }\end{array}$ \\
\hline 6 & $\begin{array}{l}\text { Capacidade de } \\
\text { Memorização }\end{array}$ & & &
\end{tabular}

\begin{tabular}{|c|c|c|c|c|}
\hline \multicolumn{5}{|c|}{ Metas decorrentes da experiência com os usuários } \\
\hline ID & Metas & Atende & Não Atende & Descrição \\
\hline 1 & Satisfatório & $X$ & & $\begin{array}{l}\text { O usuário sente-se satisfeito ao realizar uma tarefa bem } \\
\text { mais rápido do que se tivesse que ir até a instituição } \\
\text { realizá-la. }\end{array}$ \\
\hline 2 & Compensador & $\mathrm{X}$ & & Evita deslocamentos desnecessários até a instituição. \\
\hline 3 & Agradável & & $\mathrm{X}$ & $\begin{array}{l}\text { Desagradável devido aos vários problemas de usabilidade } \\
\text { e design. }\end{array}$ \\
\hline 4 & Motivador & & $\mathrm{X}$ & $\begin{array}{l}\text { As situações indesejadas que o sistema propõe, deixam o } \\
\text { usuário desmotivado a usá-lo. }\end{array}$ \\
\hline 5 & $\begin{array}{l}\text { Esteticamente } \\
\text { Apreciável }\end{array}$ & & $\mathrm{X}$ & $\begin{array}{l}\text { Má combinação de cores, fontes pouco legível, } \\
\text { informações desnecessárias não são transparentes ao } \\
\text { usuário. }\end{array}$ \\
\hline 6 & $\begin{array}{l}\text { Emocionalmente } \\
\text { Adequado }\end{array}$ & & $\mathrm{X}$ & $\begin{array}{l}\text { O público alvo é em sua maioria jovem, por isso, querem } \\
\text { acessar suas informações da maneira mais fácil e rápida } \\
\text { possível, utilizando um ambiente com uma interface } \\
\text { bonita e agradável. }\end{array}$ \\
\hline
\end{tabular}


Os resultados da análise mostram que o sistema é útil e compensador levando em consideração que os processos educacionais que antes eram manuais agora são automatizados, porém, do ponto de vista das tecnologias interativas centradas no usuário a interface apresenta falhas que podem torná-lo pouco prazeroso ou até de difícil utilização e memorização.

Ao final do levantamento dos princípios e metas de usabilidade com alunos e servidores foram extraídos os seguintes requisitos e atributos de usabilidade:

- Facilidade e Rapidez na interação com alunos e servidores - A interface do sistema de comunicação e interação deve ser fácil e rápido de aprender e utilizar;

- Organização das Informações - O sistema deve prover interação objetiva, fornecendo ao usuário níveis de informações necessárias ao objetivo da tarefa pretendida.

- Agradável e Geração de Satisfação - O sistema deve prover um ambiente com interface agradável e que satisfaça aos requisitos do usuário;

- Eficiência - O sistema deve gerar em sua interface facilidade de memorização, aprendizado, segurança e visualização dos objetos do design.

\subsection{Prototipação}

Inicialmente nesta etapa do projeto foi utilizada a técnica de prototipagem rápida em papel (paper prototype), após essa fase foram utilizadas as ferramentas Balsamiq e Pencil para prototipação em média fidelidade no intuito de aperfeiçoar os protótipos recém criados em papel. Por fim foi utilizada a ferramenta Bootstrap para desenvolver as interfaces de forma que permitam uma experiência de visualização e navegação em diferentes tipos de dispositivos.

Todas as interfaces das principais funções do SAGE foram prototipadas e validadas, no entanto, devido ao espaço disponível neste documento apenas algumas serão apresentadas. As Figuras 2 e 3 apresentam as seguintes funcionalidades: visibilidade, feedback, definição de identidade visual e organização das informações.

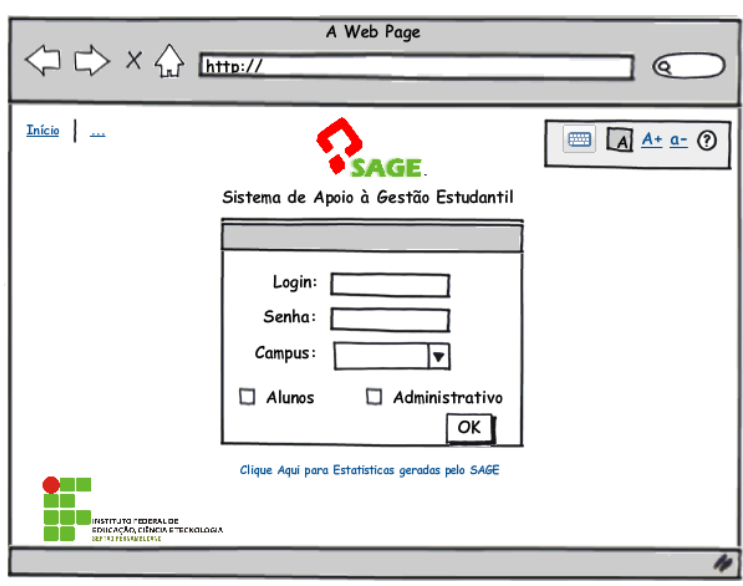

Figura 2: Página inicial de login do usuário Aluno.

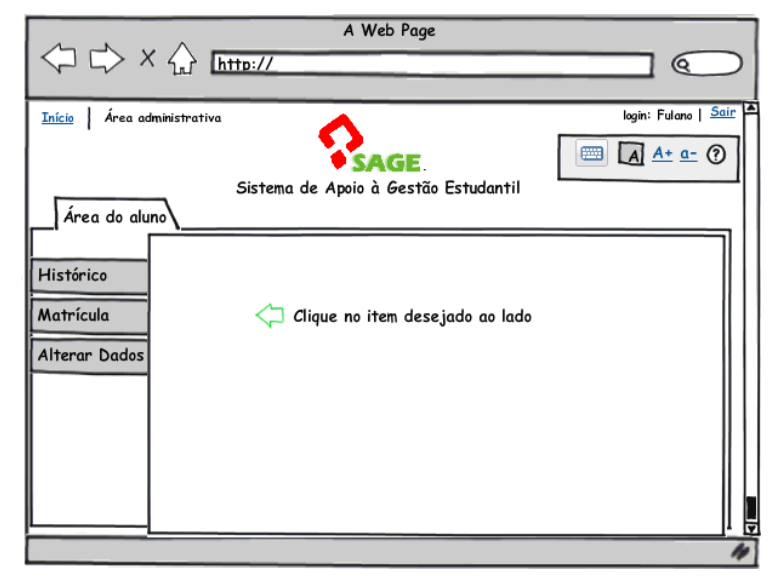

Figura 3: Página inicial do usuário Aluno. 
Os protótipos seguintes, descritos pelas Figuras 4 e 5 mostram como será definida a interação do professor com a interface proposta, com isso, são aplicados os requisitos de organização das informações e eficiência do ponto de vista da facilidade de memorização, aprendizado, segurança e visualização dos objetos do design.

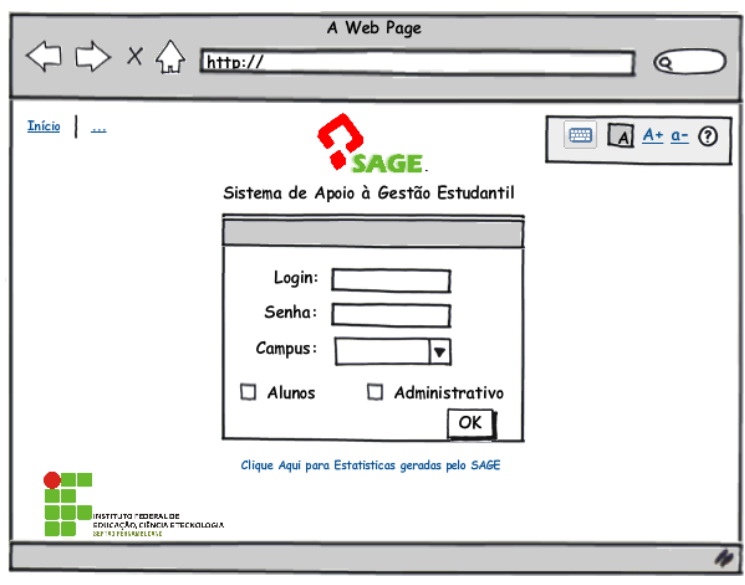

Figura 4: Página inicial de login do Professor.

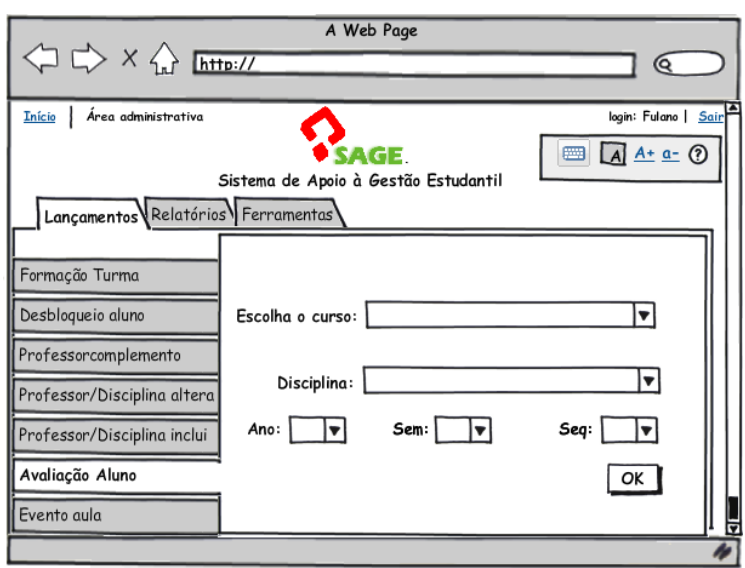

Figura 5: Página inicial de avaliação do Professor.

A seguir, através das Figuras 6, 7 e 8 são apresentados os protótipos de alta fidelidade construídos com a utilização do framework Bootstrap, que mostram uma proposta de interface responsiva das principais telas do SAGE.

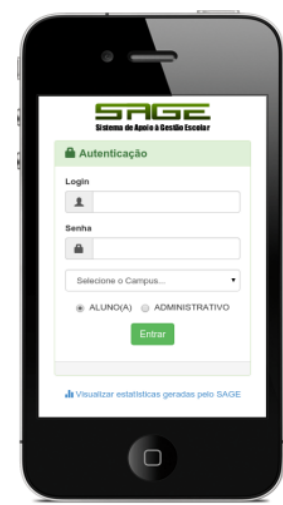

Figura 6: Página inicial de login do Aluno.

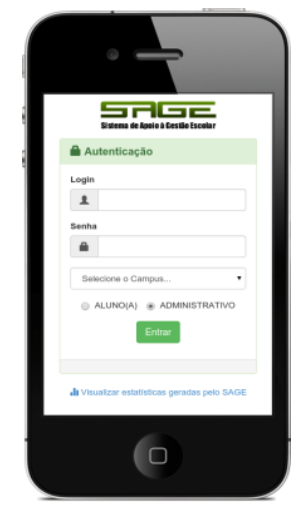

Figura 7: Página inicial de login do Professor.

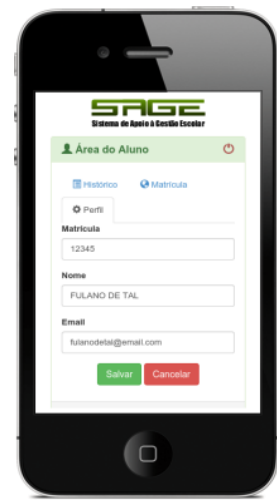

Figura 8: Página inicial do perfil do Aluno.

Após as interfaces responsivas adaptadas para smartphones serem apresentadas, o protótipo da Figura 9 mostra a tela adaptada para dispositivo do tipo tablet. 


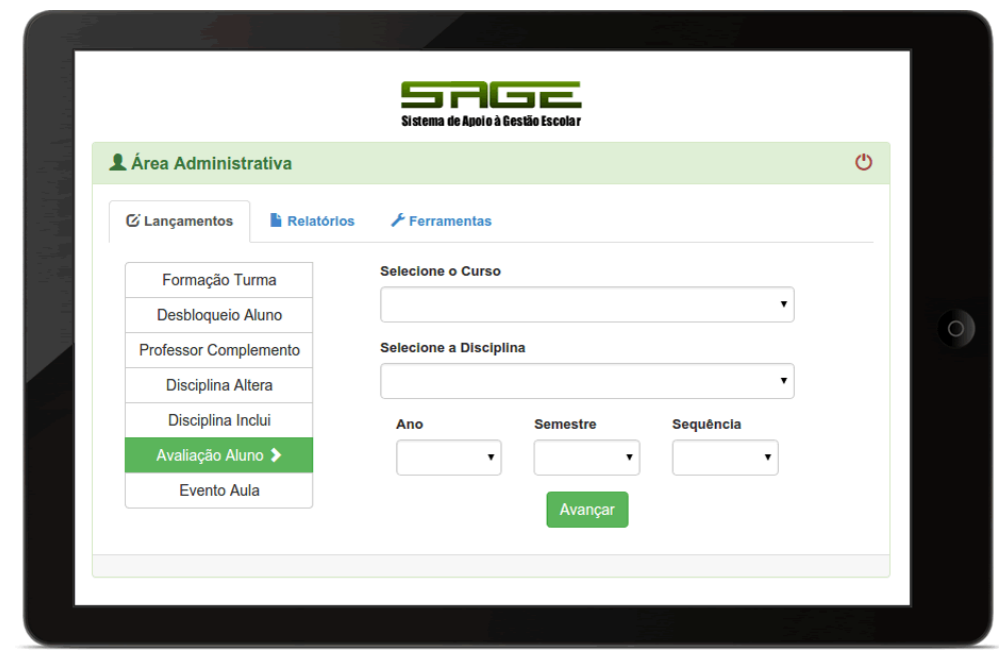

Figura 9: Página Inicial de Avaliação do Professor.

\subsection{Validação dos Protótipos}

Os testes para validação dos protótipos foram realizados com quatro usuários do SAGE (dois professores e dois alunos), com a idade entre 15 e 50 anos, dois de nível médio e dois de nível superior, mais de um ano de conhecimentos básicos de informática (uso da internet, ferramentas office, mouse e teclado). Os participantes preencheram questionários acerca do seu perfil e ao final sobre satisfação do produto utilizado.

A técnica foi aplicada por dois pesquisadores avaliadores, um aluno pesquisador simulou o funcionamento de um microcomputador, realizando as trocas de interfaces e navegação, com base nas solicitações feitas pelos usuários testados. O professor avaliador observou e preencheu, em cada sessão de teste, um formulário de coleta de dados, onde foram registrados dados sobre o desempenho do participante, número de erros encontrados, sucesso das tarefas e detalhes observados durante a execução de cada tarefa. O papel de cada pesquisador, dinâmica dos testes e os motivos para realização deste foram antecipadamente explicados para cada usuário de teste participante deste processo.

Após a finalização dos testes dos protótipos de baixa fidelidade com usuários, a análise de seus resultados foi positiva, pois, apesar dos protótipos apresentados aos usuários conter um visual diferente daquele (SAGE) que o usuário está acostumado a utilizar, todos se adaptaram às novas funcionalidades do sistema, utilizando devidamente seus novos recursos de forma simples, fácil e eficiente.

\section{Conclusão}

A interface atual do Sistema de Apoio à Gestão Escolar - SAGE apresenta falhas em relação à usabilidade e adaptabilidade, estas podem deixar o usuário constrangido e/ou confuso, ou até mesmo induzi-lo ao erro. Através da pesquisa apresentada, foi desenvolvida uma nova proposta de interface. Foram criados e testados protótipos de baixa, média e alta fidelidade para validar o novo design sugerido, permitindo que se 
viabilize entre alunos e servidores interação fácil, organizada, rápida e eficiente, facilitando a satisfação dos usuários do sistema, inclusive de forma responsiva.

A proposta de nova interface permite um resultado final satisfatório, possibilitando aos envolvidos no projeto a capacidade de identificar e resolver problemas de usabilidade e adaptabilidade de softwares web. A pesquisa permite novas discussões técnico-científicas entre desenvolvedores de software e usuários sobre a importância da utilização de técnicas de adaptabilidade e usabilidade web, além de proporcionar estímulos ao interesse pela pesquisa científica por parte da comunidade acadêmica. Os próximos passos envolvem a implementação funcional dos protótipos e interfaces criadas visando à integração desta proposta à interface atual do SAGE.

\section{Referências}

[1] Cybis, Abreu, W., Betiol, A. H., \& Faust, R. (2007). Ergonomia e usabilidade: conhecimentos, métodos e aplicações. Novatec Editora.

[2] Marcotte, E. (2011). Responsive web design. Editions Eyrolles.

[3] Nielsen, J. (1990). Evaluating hypertext usability. In Designing hypermedia for learning (pp. 147-168). Springer Berlin Heidelberg.

[4] Preece, J. Rogers, Y. e Sharp, H. (2005) Design de Interação: Além da Interação Humano-Computador. Bookman.

[5] Pressman, R. S. (2011). Engenharia de software. McGraw Hill Brasil.

[6] Kimura, M. H., Kemczinski, A., Gasparini, I., Pernas, A. M., Pimenta, M. S., \& de Oliveira, J. P. M. (2012). Aumentando a Flexibilidade de um Sistema e-learning Adaptativo através da Abordagem Responsive Webdesign. IEEE-RITA, 7(4), 203210.

[7] Sommerville, I. (2003). Engenharia de software (Vol. 6). Addison Wesley.

[8] Schmitz, D. (2014). Bootstrap 3: Framework front-end para desenvolvimento web e mobile. Leanpub.

[9] Silva, M. S. Web Design Responsivo. Novatec, 2014.

[10] Zemel, T. Web Design Responsivo: Páginas adaptáveis para todos os dispositivos. Casa do Código, 2012. 